\title{
Widely Tunable Wavelength-Selective Reflector Using Polymer Waveguide Double-Ring-Resonator Add/Drop Filter and Loop-back Mirror
}

\author{
Ho Lee, Gun-Woo Kim, Jun-Oh Park, Su-Hyun Kim, and Youngchul Chung* \\ Department of Electronics and Communications Engineering, Kwangwoon University, Seoul, \\ 139-701, Korea
}

(Received August 5, 2008 : revised September 1, 2008 : accepted September 1, 2008)

\begin{abstract}
A wavelength-selective reflector, composed of a double-ring-resonator add/drop filter and a loop-back mirror at the drop port to be used for a hybrid-integrated tunable laser is designed and fabricated. High-index-contrast polymer waveguide is used to realize a compact device as small as $2 \mathrm{~mm} \times 0.4 \mathrm{~mm}$. The radii of the rings are slightly different from each other to obtain a wide wavelength tuning via a vernier effect. Peak reflectivity is measured to be $52 \%$ for TE mode and polarization-dependent wavelength shift is $0.95 \mathrm{~nm}$. The tuning range is measured to be about 42 $\mathrm{nm}$ for applied current as small as $46 \mathrm{~mA}$.
\end{abstract}

Keywords : Ring resonators, add/drop filters, polymer waveguides, wavelength-selective reflectors, tunable lasers

OCIS codes : (130.0130) Integrated optics; (250.5460) Polymer waveguides-fibers; (230.7390) Waveguides, planar; (120.2440) Filters; (230.5750) Resonators

\section{INTRODUCTION}

Since a ring resonator was proposed by Marcatili in 1969 [1], it has been a popular research subject because it is expected to be used as a basic device for a variety of large scale functional photonic integrated circuits used in optical networks. These circuits have high complexity and many functions. In practice, ring resonators have been used to implement many optical devices such as optical add/drop filters, tunable lasers, wavelength reflectors, optical switches, and bio sensors [2-8]. Meanwhile, a study for a low-cost wavelength tunable source in a wavelength-division-multiplexing passive optical network (WDM-PON) has been recently researched by many research teams to realize a stable and economical high-capacity PON system [9-10]. Another promising light source for WDM-PON called wavelength-locked FP-LD has some problems such that its performances depend on injected power and the modulation rate is limited. Therefore a wavelength-tunable source is considered to be an ultimate solution for WDM-PON, and low-cost tunable sources have been actively sought [11].

*Corresponding author: ychung@kw.ac.kr
One of the ways to implement a low-cost tunable source is to use a hybrid integration of a reflective semiconductor optical amplifier with a wavelengthselective reflector based on waveguide structures. A grating structure engraved on a polymer waveguide [11-12] or a coupled-ring reflector [3] has been employed to realize a wavelength-selective tunable reflective filter for hybrid integration. The fabrication of the grating reflector needs an additional process of engraving after the waveguide formation, which might increase the cost of the device. The coupled-ring reflector has been recently introduced to simplify the fabrication process of the wavelength-selective reflector and the feasibility has been given through the experimental works [8]. The coupled-ring reflector is composed of two coupled rings both of which are coupled to a bus waveguide. Therefore the couplings from the bus waveguide and the coupled rings and vice versa result in a reflection naturally. A double-ring add/drop filter is also a good candidate for the transmission-type wavelength filter, and it is expected to provide better wavelength-selectivity than the coupled-ring reflector if a reflector is made by placing a mirror at the drop port. A mirror can be pro- 
vided by coating the drop port facet, but this requires an additional process. To provide fabrication simplicity and enhanced wavelength selectivity for the wavelength -selective reflector, a double-ring resonator add/drop filter integrated with a loop-back mirror at the drop port is proposed and the device characteristics are investigated through fabrication and measurement results. In this paper, a brief principle of the proposed wavelength -selective reflector is mentioned in section II, its design and fabrication in section III, measurement results IV, and finally conclusions in section $\mathrm{V}$.

\section{PRINCIPLE}

As depicted in Fig. 1, the proposed wavelength-selective reflector is composed of a double-ring add/drop filter and a loop-back mirror at the drop port. The brief operation principle is as follows. An incident wave is coupled into the first ring resonator as the clockwise wave through a bus-ring coupler $\left(\kappa_{0}\right)$. This wave is coupled into the second ring resonator as the counterclockwise wave through a ring-ring coupler $\left(\kappa_{1}\right)$, and then this wave is coupled into another bus waveguide through ring-bus coupler $\left(\kappa_{0}\right)$. In this coupling process, we keep in mind that the waves at the wavelengths that meet the resonant condition for both ring resonators can be transferred into the drop port. The loop-back mirror positioned at the drop port provides the reflection of the dropped wavelength components. In particular, if two ring resonators are designed to have slightly different radii, the FSRs (free spectral ranges) and resonant wavelength of two resonators are slightly different from each other. Therefore, the strong reflectivity is obtained only at particular wavelengths at which two rings are simultaneously resonant. Slightly different radii between two ring resonators have an important role for the wavelength tuning characteristic in wide range using a vernier effect. In other words, the slight tuning of the resonant wavelength in one of the rings causes the tuning of the drop wavelength over one FSR, which greatly enhance the tuning range. The tuning range is given by

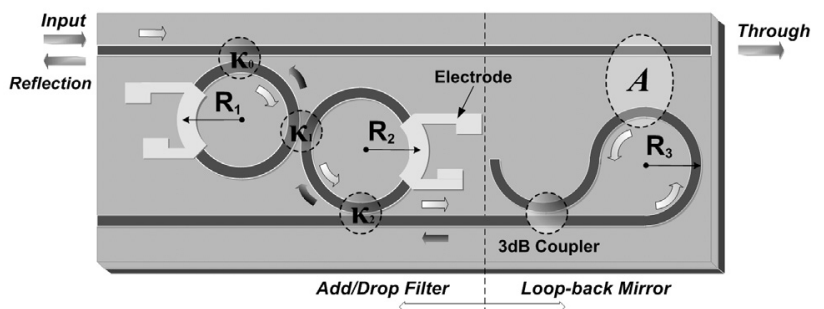

FIG. 1. Schematic configuration of the proposed wave length-selective reflector.

$$
\Delta \lambda_{\text {tune }}=\frac{\lambda_{0}^{2}}{2 \pi n_{g}\left(R_{2}-R_{1}\right)}=F \cdot \Delta \lambda_{R 2}
$$

where $\lambda_{0}$ represents a center wavelength, $n_{g}$ is group index at specific wavelength, and $R_{1}$ and $R_{2}$ are radii of two rings. $\triangle \lambda_{R 2}$ is the FSR of ring 2 and $F$ is a tuning enhancement factor. In the case of our device, $\lambda_{0}$ is $1550 \mathrm{~nm}, n_{g}$ is 1.447 at a center wavelength, and $\mathrm{R}_{1}$ and $\mathrm{R}_{2}$ are $150 \mu \mathrm{m}$ and $156 \mu \mathrm{m}$, respectively. Therefore, the tuning range is calculated to be about $44 \mathrm{~nm}$.

The tradeoff between the tuning enhancement and the wavelength selectivity, however, has to be made. The device with large radius difference between two resonators has higher difference between FSRs than that with small radius difference, and can provide a superior wavelength selectivity characteristic. Meanwhile, in the case of the tuning characteristic, the tuning range for a large radius difference between two resonators is narrower than that for a small radius difference.

\section{DESIGN AND FABRICATION}

The polymer waveguide cross-section is shown in Fig. 2. To realize a compact size, we use a polymer material with high-index-contrast as much as $8.7 \%$ and the buried waveguide has a core whose width and height are $1.5 \mu \mathrm{m}$ and $1.6 \mu \mathrm{m}$, respectively. Meanwhile, the coupling ratios of bus-ring coupler and ring-ring coupler are designed to be 0.4 and 0.09 , respectively. But there is a possibility of fabrication errors, and the dimensions considered during the design process may not be obtained. Thus we have designed the directional couplers whose coupling ratios are maintained even if fabrication errors are generated. When the waveguide's width changes from $1.1 \mu \mathrm{m}$ to $1.9 \mu \mathrm{m}$ the intensity of mode of the waveguide is shown in Fig. 3. It can be seen that the intensity of modes does not change when a distance from the center of the waveguide is more than $2.3 \mu \mathrm{m}$. According to this fact, when a gap between the centers of the waveguides is more than $2.3 \mu \mathrm{m}$, it is expected to get directional couplers with the fabrication-errortolerant characteristic. In Fig. 4, the bus-ring coupling ratio versus ring-ring coupling ratio satisfying maximallyflat condition of the ADF is shown [2][13-14]. When the

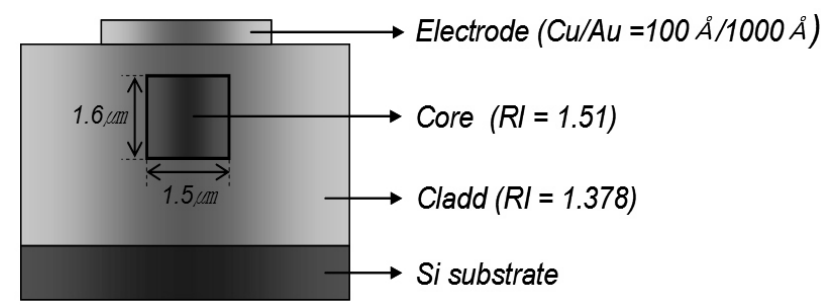

FIG. 2. Cross-section of the waveguide. 


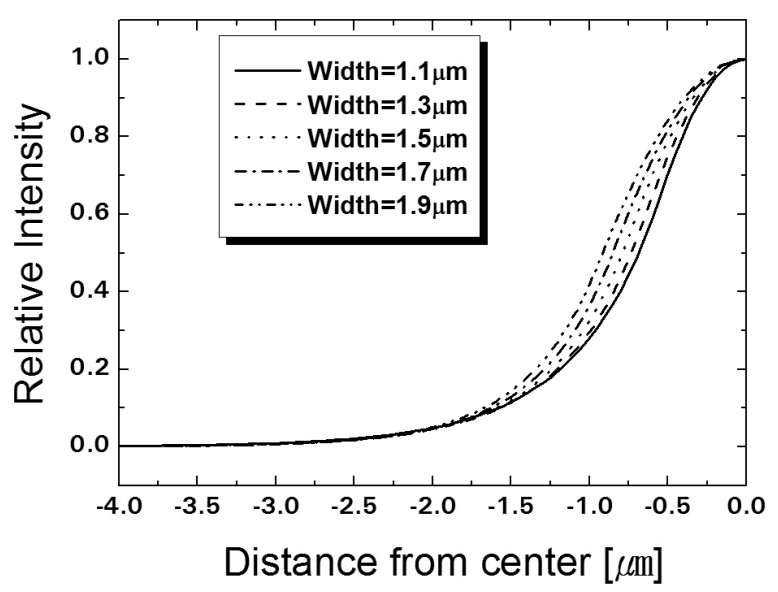

FIG. 3. Mode profiles as the waveguide width changes from $1.1 \mu \mathrm{m}$ to $1.9 \mu \mathrm{m}$.

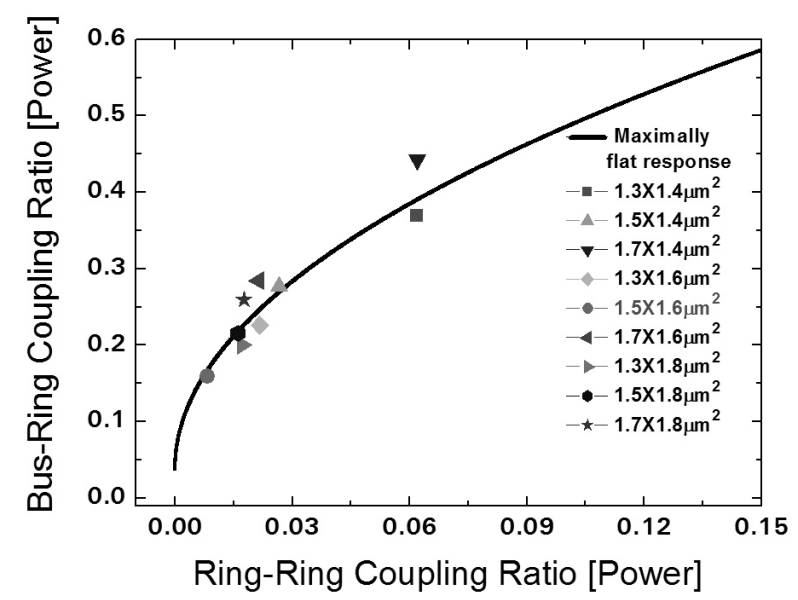

FIG. 4. Coupling ratio pairs satisfying maximally-flat condition.

condition is not satisfied, the transmission spectrum would show split peaks or the peak reflectivity smaller than one even for a lossless case. The above-mentioned coupling ratio pairs (bus-ring and ring-ring coupling ratios) are calculated using three-dimensional beam propagation method assuming that there are fabrication errors in the waveguide dimensions, and plotted as data points in Fig. 4. As you can see, if the error range of width and height of the waveguide is within $\pm 0.2 \mu \mathrm{m}$, the positions of ring-ring and bus-ring coupling ratio pairs are close to the maximally-flat-condition. The fabrication error can be controlled to be smaller than $\pm 0.2 \mu \mathrm{m}$ using standard processing equipments and it is believed that the proposed device would show an excellent errortolerant characteristic.

To get good characteristics from the proposed reflector, we must design the loop-back mirror carefully. The reflector can provide high reflectivity if the loop-back mirror has low loss and high reflection in a wide wavelength range. So, an optimized design for the $3 \mathrm{~dB}$ cou-

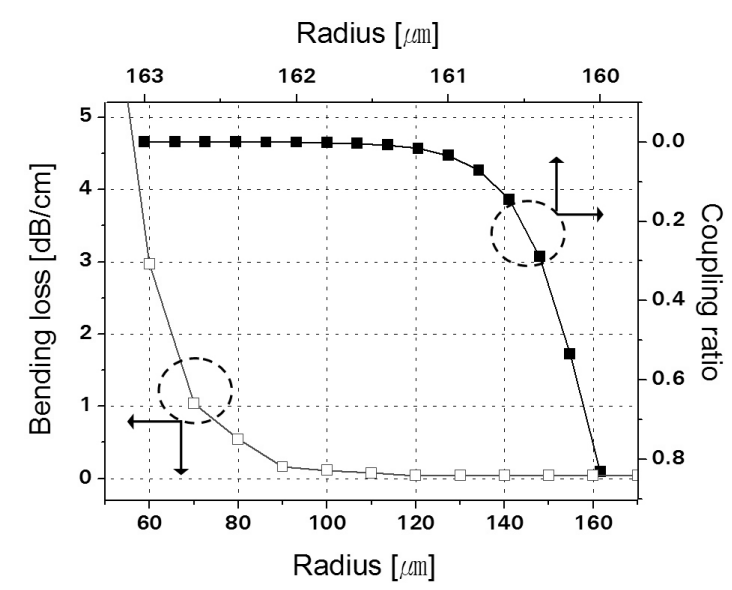

FIG. 5. Bending loss and coupling ratio as a function of radius.

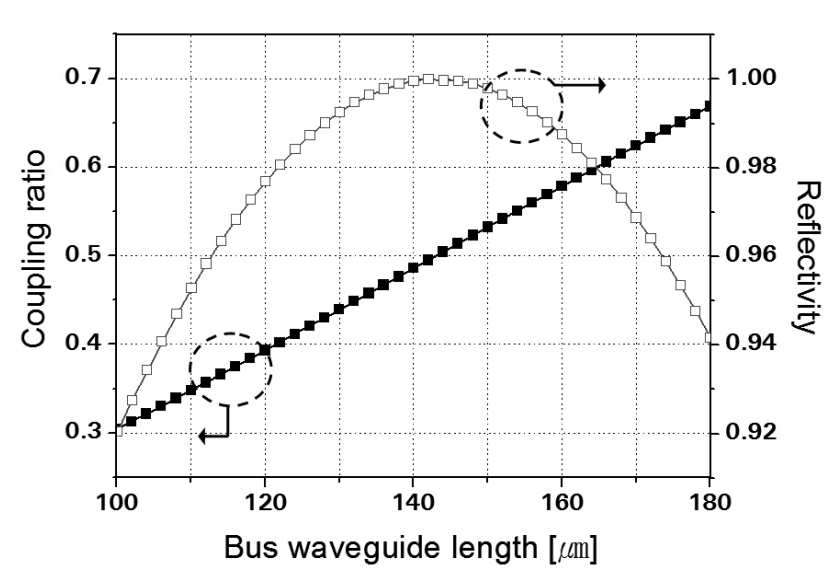

FIG. 6. Coupling ratio and reflectivity as a function of inserted bus waveguide length.

pler and bent-waveguide which are key components of the loop-back mirror is needed for good characteristics of our reflector. In bent waveguide design, we calculate the bending loss and coupling ratio in the region $\mathrm{A}$ of Fig. 1, and the result is shown in Fig. 5. As can be seen, the normalized bending loss for the radius larger than $120 \mu \mathrm{m}$ is smaller than $0.005 \mathrm{~dB}$. Note that if there is coupling effect in the region $\mathrm{A}$, it interferes with normal reflection operation. So, we choose the optimum radius to be $150 \mu \mathrm{m}$. In Fig. 6 we show the power coupling ratio of the $3 \mathrm{~dB}$ coupler and the reflectivity of the loop-back mirror as a function of the bus waveguide length inserted into the $3 \mathrm{~dB}$ coupler to precisely control the coupling ratio. When the bus waveguide length is $141.85 \mu \mathrm{m}$, the power coupling ratio is 0.5 and therefore the reflectivity is unity. When the waveguide width and height error is within $\pm 0.2 \mu \mathrm{m}$, the coupling ratio of the $3 \mathrm{~dB}$ coupler could deteriorate to about \pm 0.25 . Even with this error the reflectivity can be maintained to be over $90 \%$. 
As stated above, the reflector is fabricated as a planar lightwave circuit using polymer material to take advantage of simple fabrication process and low cost. The fabrication process is as follows. Firstly, a $12 \mu \mathrm{m}$-thick bottom cladding is formed using spin coating of polymer resin named LFR-378 (product of Chem-optics) on the Si substrate followed by thermal and UV curing. The waveguide pattern is formed by photo-lithography and inductively coupled plasma (ICP) etching process. Then the polymer resin named WIR30-510 is deposited and the curing process is carried out to make the core layer. The core layer is covered by the $5.3 \mu \mathrm{m}$-thick upper cladding layer. Finally heating electrodes are formed by evaporating $\mathrm{Cr}(100 \AA)$ and $\mathrm{Au}(1000 \AA)$. Finally, the each facet was polished after cleaving the device.

\section{MEASUREMENT RESULTS}

The micro-photograph of the fabricated wavelengthselective reflector is shown in Fig. 7. The footprint of the device is as compact as $2 \mathrm{~mm} \times 0.4 \mathrm{~mm}$. The busring and ring-ring coupling ratios are designed to be 0.4 and 0.09 , respectively. Optical responses at the through port for each polarization are shown in Fig. 8 . Strong rejection at the wavelength of $1594 \mathrm{~nm}$ and $1593.05 \mathrm{~nm}$ for TE and TM polarization, respectively, is observed. In other words, polarization-dependent wavelength shift of this device is $0.95 \mathrm{~nm}$. The reflection spectrum for each polarization is shown in Fig. 9. As can be seen, the free-spectral-range of the reflector

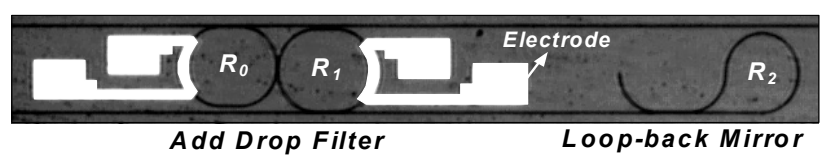

FIG. 7. Micro-photograph of the fabricated wavelength-selective reflector.

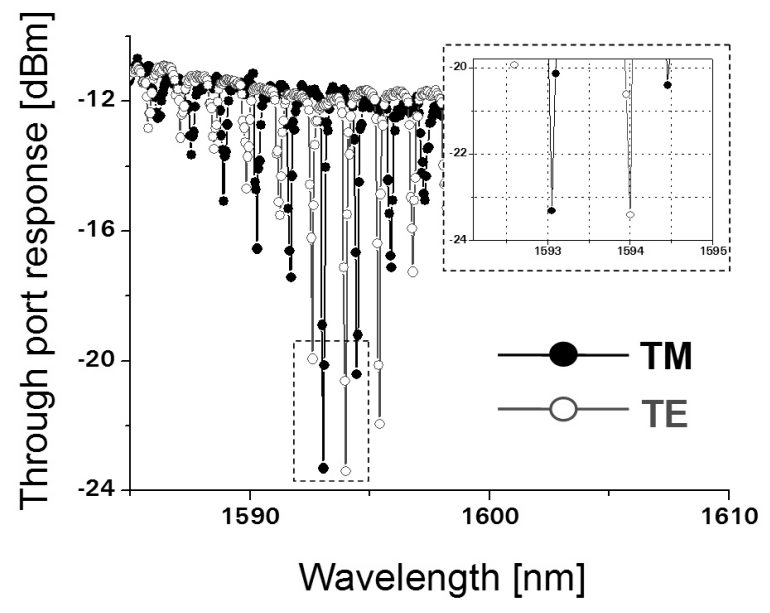

FIG. 8. Optical responses at the through port. is $1.44 \mathrm{~nm}$ which is close to the design value. It is observed that high reflections are observed at the same wavelength where strong rejections appear at the through port. It is also observed that the smaller the reflection peak becomes the farther the wavelength is from the peak reflection wavelength. This happens because the resonant wavelengths of two rings get mismatched further as the wavelength gets distant from the peak reflection wavelength. As can be seen in Fig. 9(a), for TE polarization, the reflectivity is $52 \%$ and full width at half maximum (FWHM) bandwidth is $0.13 \mathrm{~nm}$. But for TM polarization, the reflectivity and FWHM bandwidth are $42 \%$ and $0.14 \mathrm{~nm}$, respectively. In the aspect of the wavelength selectivity, TE polarization is 4 times better than TM polarization by comparing Fig. 9(a) and (b), probably because the coupler characteristics for TE mode is closer to the design values than for TM mode.

When certain amount of current is applied to the electrode on top of one of two ring resonators, the refractive index (RI) of the ring is adjusted while the $\mathrm{RI}$ of another is maintained. Therefore, with the supply of proper amount of the current the aligned wavelength can be shifted one FSR by one FSR discretely. The

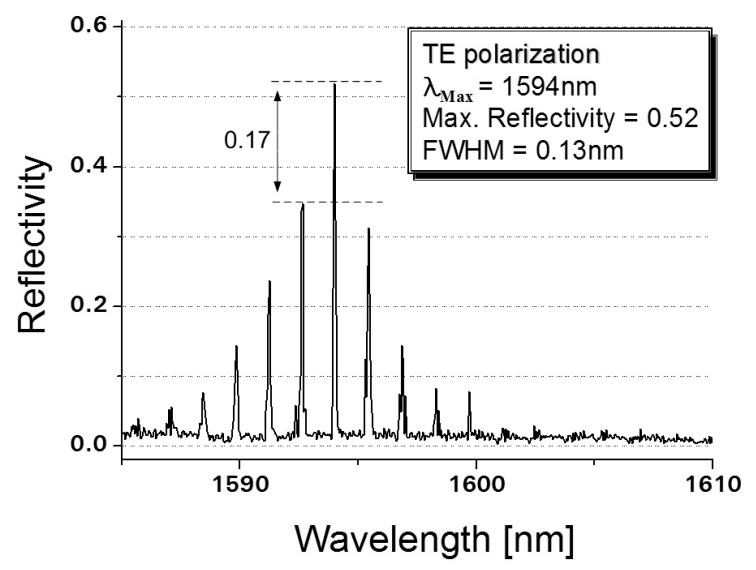

FIG. 9. Reflectivity spectra for (a) TE polarization

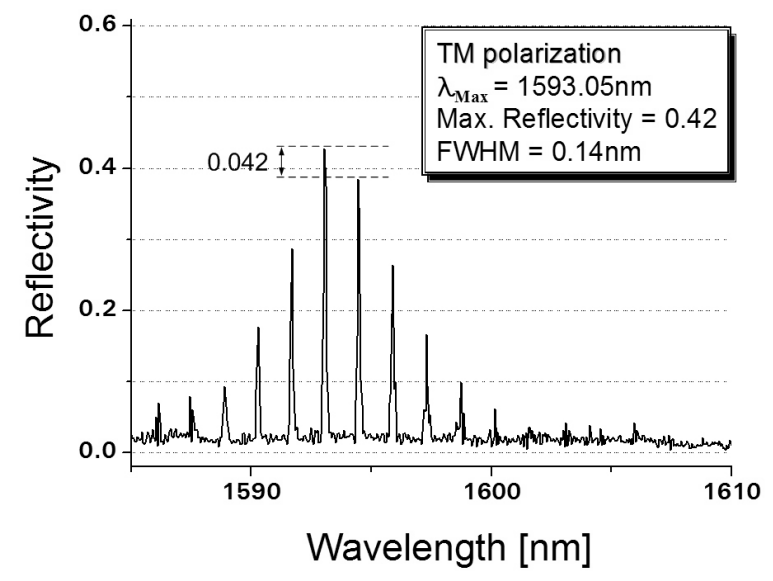

(b) TM polarization. 


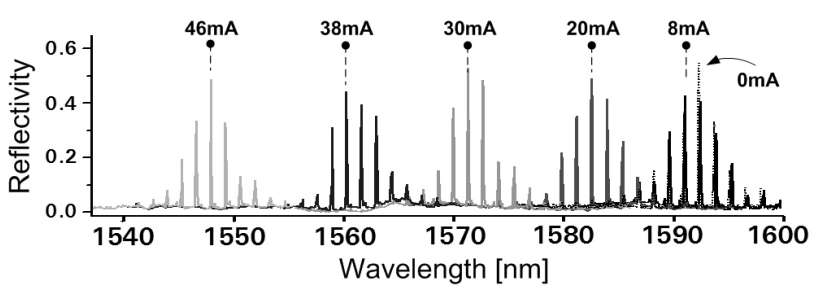

FIG. 10. The wavelength tuning characteristic.

measured wavelength tuning spectra of the reflector are shown in Fig. 10. The tuning range is about $42 \mathrm{~nm}$ for the tuning current as small as $46 \mathrm{~mA}$ corresponding to the tuning power of $22 \mathrm{~mW}$, which is similar to the theoretical tuning range. This wavelength-selective reflector is especially useful in realizing a widely tunable laser by hybrid-integrating it with a reflective semiconductor optical amplifier.

\section{CONCLUSION}

A new wavelength-selective reflector with high reflectivity and wide tuning characteristic has been demonstrated. The reflector is composed of a double-ring resonator add/drop filter connected with a loop-back mirror at the drop port. Use of high-index-contrast polymer material as a planar lightwave circuit platform makes it possible to realize a compact device as small as $2 \mathrm{~mm}$ $\mathrm{x} 0.4 \mathrm{~mm}$ using a simple fabrication process and possibly at a low cost. Measurement results show that maximum reflectivity is $52 \%$ with $0.13 \mathrm{~nm}$ FWHM bandwidth for TE mode. Polarization-dependent wavelength shift of the device is about $0.95 \mathrm{~nm}$. The ring radii of two rings are designed to be slightly different from each other to provide a wide tuning by a vernier effect. The ring radius of one ring is $150 \mu \mathrm{m}$ and that of the other is $156 \mu \mathrm{m}$. In this case the wavelength tuning range is about 42 $\mathrm{nm}$ for the tuning current as small as $46 \mathrm{~mA}$, which is quite similar to the theoretical value. When this wavelength-selective reflector is hybrid-integrated with a reflective semiconductor optical amplifier, a low cost tunable source for WDM-PON could be realized.

\section{ACKNOWLEDGMENT}

This work was supported by grant No.R01-2006-00010751-0 from the Basic Research Program of the Korea Science \& Engineering Foundation. This work was also partly supported by the Research Grant of Kwangwoon University in 2008.

\section{REFERENCES}

[1] E. A. J. Marcatili, "Bends in optical dielectric guides," The Bell System Technical Journal, vol. 48, pp. 21032132, 1969.

[2] B. E. Little, S. T. Chu, H. A Haus, J. Foresi, and J. -P. Laine, "Microring Resonator Channel Dropping Filters," J. Lightwave Technol., vol. 15, no. 6, pp. 998-1005, 1997.

[3] Youngchul Chung, Doo-Gun Kim, and Nadir Dagli, "Reflection properties of coupled-ring reflectors," J. Lightwave Technol., vol. 24, no. 4, pp. 1865-1874, 2006.

[4] Bin Liu, Ali Shakouri, and John E. Bowers, "Wide Tunable Double Ring Resonator Coupled Lasers," IEEE Photon. Technol. Lett., vol. 14, no. 5, pp. 600-602, 2002.

[5] Payam Rabiei and Wililam H. Steier, "Tunable Polymer Double Micro-Ring Filter,” IEEE Photon. Technol. Lett., vol. 15, no. 9, pp. 1255-1257, 2003.

[6] V. Van T. A. Ibrahim, K. Ritter, P. P. Absil, F. G. Johnson, R. Grover, J. Goldbar, and P. T. Ho, “Alloptical nonlinear switching in GaAs-AlGaAs microring resonator," IEEE Photon. Technol. Lett., vol. 14, no. 1, pp. 74-76, 2002.

[7] Y. Deki, T. Hatanaka, M. Takahashi, T. Takeuchi, S. Watanabe, T. Miyazaki, M. Horie, and H. Yamazaki, "Wide-wavelength tunable lasers with $100 \mathrm{GHz}$ FSR ring resonator," Electronic. Lett., vol. 43, no. 4, 2007.

[8] Joonoh Park, Taehyung Lee, Donghyun Lee, Suhyun Kim, Woonglin Hwang, and Youngchul Chung, "Widely Tunable Coupled-Ring-Reflector Filter Based on Planar Polymer Waveguide," IEEE Photon. Technol. Lett., vol. 20, no. 12, pp. 988-990, 2008.

[9] Geun-Young Kim and Jin-Hee Kim, "Wavelength Division Multiplexing-Passive Optical Network Based FTTH Field Trial Test," J. Opt. Soc. Korea, vol. 11, no. 3, pp. 101-107, 2007.

[10] Min-Hwan Kim,Sang-Mook Lee,Sil-Gu Mun, and Chang -Hee Lee, "A $240 \mathrm{~km}$ Reach DWDM-PON of 8-Gb/s Capacity using an Optical Amplifier," J. Opt. Soc. Korea, vol. 11, no 3, pp. 93-96, 2007.

[11] Geon Jeong, Jie-Hyun Lee, Mahn Yong Park, Cheol Young Kim, Seung-Hyun Cho, Wooram Lee, and Byoung Whi Kim, "Over 26-nm Wavelength Tunable External Cavity Laser Based on Polymer Waveguide Platform for WDM Access Networks," IEEE Photon. Technol. Lett., vol. 18, no. 20, pp. 2102-2104, 2006.

[12] Min-Suk Kwon and Sang-Yung Shin, “Tunable polymer waveguide notch filter using a thermooptic long-period grating," IEEE Photon. Technol. Lett., vol. 17, no. 1, pp. 145-147, 2005.

[13] D. Lee, T. Lee, J. Park, S. Kim, W. Hwang, and Y. Chung, "Widely Tunable double-ring-resonator add/drop filter using high-index-contrast polymer waveguide," in Cleo/Pacific Rim 2007, pp. 1-2, Aug. 2007.

[14] Dong-Hyun Lee, Tae-Hyung Lee, Jun-Oh Park, SuHyun Kim, and Youngchul Chung, "Widely Tunable Double-Ring-Resonator Add/Drop Filter," Hankook Kwanghak Hoeji, vol. 18, no. 3, June 2007. 